\title{
Pioglitazone Ameliorates Intracerebral Insulin Resistance and Tau-protein Hyperphosphorylation in Rats with Type 2 Diabetes
}

Authors

Affiliation

\author{
S.-H. Hu, T. Jiang, S.-S. Yang, Y. Yang
}

Department of Endocrinology, Tongji Hospital, Tongji Medical College, Huazhong University of Science and Technology, China

\begin{abstract}
Key words
- Alzheimer's disease

- dementia

- glucose metabolism

anti-diabetic drug
\end{abstract}

received $\quad 13.09 .2012$

first decision 13.12.2012

accepted $\quad 02.01 .2013$

\section{Bibliography}

DOI http://dx.doi.org/

10.1055/s-0032-1333277

Published online:

March 19, 2013

Exp Clin Endocrinol Diabetes

2013; 121: 220-224

(C) J. A. Barth Verlag in

Georg Thieme Verlag KG

Stuttgart · New York

ISSN 0947-7349

\section{Correspondence}

\section{Y. Yang}

Department of Endocrinology

Tongji Hospital

Tongji Medical College

Huazhong University of Science

and Technology

Wuhan 430030

China

Tel.: + 86/139/71285201

Fax: + 86/21/6408 5875

yangyandoc@yahoo.com.cn

\section{Abstract}

$\nabla$

Objective: To investigate intracerebral insulin resistance and its relationship with tau-protein hyperphosphorylation.

Methods: A rat model of type 2 diabetes (T2D) was established with streptozotocin (STZ). Diabetic rats received intragastric administration of pioglitazone (PIO group) or normal saline (T2D group) for 4 weeks. As a control, non-diabetic rats received intragastric normal saline (CTL group). The insulin concentrations in cerebrospinal fluid (CSF) and blood were determined with radioimmunoassay, and blood glucose concentration was determined using a glucose oxidation technique. Total and phosphorylated levels of protein kinase B (AKT), glycogen synthase

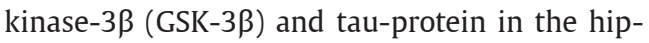
pocampus were analyzed using western blotting. Results: The plasma insulin level in the T2D group was higher, and the CSF insulin level in the

\section{Introduction}

$\nabla$

Diabetes is the most common endocrine-metabolic disease, and the prevalence of type 2 diabetes (T2D) in China has now reached levels of $9.7 \%$ in people aged more than 20 years old [1]. With improvements in therapy, the lifespans of individuals with diabetes have been prolonged, such that cognitive disorders and dementia (Alzheimer's disease, AD) have become more common, important complications. Indeed, epidemiologic data suggest that the risk of developing dementia is 1.4-4.3 times higher in people with T2D than in non-diabetic individuals [2-4].

The main clinical characteristic of $\mathrm{AD}$ is a progressive impairment of memory. The major pathologic features of AD are intraneuronal neurofibrillary tangles, comprised of paired helical filaments (PHF), that arise as the consequence of hyperphosphorylation of a microtubule-associ-
T2D group lower than in the CTL group. Hippocampal phosphorylated AKT and phosphorylated GSK-3 $\beta$ levels were significantly lower in the T2D group than in the CTL group. Hippocampal tauprotein in the T2D group was hyperphosphorylated at Ser199 and Ser396. Plasma insulin levels in the PIO group were lower than in the T2D group, with no differences in CSF insulin levels. Phosphorylated AKT and phosphorylated GSK-3 $\beta$ levels in the PIO group were significantly higher than in the T2D group. Hippocampal phosphorylated tau-protein (Ser199/Ser396) was lower in the PIO group than in the T2D group. Conclusion: Hyperphosphorylation of tau-protein in pioglitazone-treated rats with T2D was improved. Rats with T2D have both cerebral insulin resistance and cerebral hypoinsulinism. Pioglitazone can ameliorate intracerebral insulin resistance and decrease tau-protein hyperphosphorylation, but cannot increase intracerebral insulin levels.

ated protein (tau-protein), and senile plaques that result from the accumulation of amyloid $\beta$ (Aß) [5]. Tau-protein hyperphosphorylation is associated with brain insulin deficiency or disorders of insulin signal transduction [6].

Unlike its action in peripheral tissues, insulin has only limited effects on glucose metabolism in the central nervous system. However, insulin does have important effects on neuronal function, including neurotransmitter release and reuptake, neuronal synaptic plasticity, learning and memory [7]. Studies have suggested that plasma insulin levels in patients with AD are normal, but that cerebrospinal fluid (CSF) insulin levels are decreased [8]. This is thought to be due to an impairment of insulin transport across the blood-brain-barrier (BBB) of patients with $\mathrm{AD}$, since it is known that intracerebral insulin is derived from circulating blood [9]. Consistent with this, intracerebroventricular injection of 
insulin has been reported to enhance memory in rats, tested in a passive-avoidance task experiment [10]. Furthermore, insulin deficiency exacerbates cerebral amyloidosis and behavioral deficits in a transgenic mouse model of $\mathrm{AD}$ [11]. Together, these studies suggest that brain insulin deficiency is one of the risk factors for AD. However, non-diabetic patients with $A D$ have been reported to show intracerebral insulin resistance [12]. Intracerebral insulin resistance is analogous to the situation of peripheral insulin resistance, and reflects reduced actions of insulin in the central nervous system $[13,14]$. Decreased activation of intracerebral insulin signaling cascades leads to the impairment of the normal functions of insulin in neurons; these functions include regulation of glucose metabolism, neural growth, survival and remodeling, and microtubule assembly. Interestingly, Li et al. (2011) have identified 8 proteins that are differentially expressed in type 1 diabetes mellitus, and have suggested that these may be involved in the pathogenesis of diabetic encephalopathy [15].

T2D is characterized by insulin resistance, and is associated with brain tau-protein hyperphosphorylation and increased activity

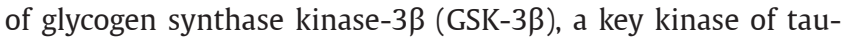
protein $[16,17]$. GSK-3 $\beta$ lies downstream of phosphatidylinositol-3-kinase (PI3K) and protein kinase B (AKT) in the insulin signal transduction cascade, and its activity is depressed by PI3K/AKT. Normal insulin signaling excites PI3K/AKT and suppresses GSK-3 $\beta$, thereby inhibiting tau-protein hyperphosphorylation. It is possible that there is not only peripheral but also intracerebral insulin resistance in T2D, leading to decreased activity of PI3K/AKT, increased activity of GSK-3 $\beta$ and tau-protein hyperphosphorylation. If this were the case, thiazolidinediones (agonists of the peroxisome proliferator-activated receptor- $\gamma$ [PPAR $\gamma$ that act as insulin sensitizers) would be expected to alleviate intracerebral insulin resistance and tau-protein hyperphosphorylation. One study has reported that pioglitazone, a thiazolidinedione anti-diabetic drug, can improve the cognitive function of patients with T2D and mild AD [18]. Furthermore, in a rat model of T2D, Kanazawa et al. (2011) reported that pioglitazone was able to reduce serum concentrations of pentosidine, consistent with a decrease in the levels of advanced glycation end products that are associated with impaired insulin function [19]. However, it is currently not clear whether CSF insulin levels in rats with T2D are decreased in a manner similar to that seen in patients with $\mathrm{AD}$.

In this study, we have established a model of T2D in rats, and investigated the effects of pioglitazone on plasma and CSF insulin levels, AKT/GSK-3 $\beta$ activity in the insulin signal transduction cascade, and phosphorylated levels of tau-protein.

\section{Materials and Methods}

$\nabla$

Rat model of T2D

Establishing T2D

21 male Sprague-Dawley (SD) rats, weighing $150-180 \mathrm{~g}$ and aged 10-12 weeks, were purchased from the Experimental Center of Tongji Medical College, Huazhong University of Science and Technology, China. Rats were assigned randomly to one of 3 groups: control group (CTL, $n=7$ ), untreated T2D group $(\mathrm{T} 2 \mathrm{D}, \mathrm{n}=7$ ) and pioglitazone-treated T2D group (PIO, $\mathrm{n}=7$ ). Rats in the T2D and PIO groups were fed with high calorie food (calorie percentage: carbohydrate $26.0 \%$, protein $15.2 \%$, fat [refined lard] $58.8 \%$ ), while those in the CTL group were fed on a stand- ard diet. After 3 months of feeding, rats in the T2D and PIO groups received intraperitoneal injection of streptozotocin $30-35 \mathrm{mg} / \mathrm{kg}$ (Sigma; streptozotocin powder dissolved in $0.1 \mathrm{~mol} / \mathrm{L}$ citrate buffer solution, $\mathrm{pH} 4.3$ ), while rats in the CTL group received intraperitoneal injection of $0.1 \mathrm{~mol} / \mathrm{L}$ citrate buffer solution. 72 hours later, blood samples were drawn from the caudal vein. Obtaining rats with a plasma glucose level $\geq 16.7 \mathrm{mmol} / \mathrm{L}$ was considered to be indicative of successful establishment of the T2D model. All rats in the T2D and PIO groups had plasma glucose levels $\geq 16.7 \mathrm{mmol} / \mathrm{L}$.

\section{Treatment with pioglitazone}

Rats in the PIO group were intragastrically administered pioglitazone $20 \mathrm{mg} / \mathrm{kg}$ (Takeda Pharmaceutical Co.) for 4 weeks, while those in the T2D and CTL groups were intragastrically administered normal saline for 4 weeks. The dosage of pioglitazone chosen was based on that used in previous studies [20]. 3 days after the end of this 4-week administration period, the surviving rats were sacrificed by cervical dislocation; survival was $7 / 7$ in the CTL group (100\%), 5/7 in the TD2 group (71.4\%) and 6/7 in the PIO group (85.7\%). Throughout the experimental period, all rats were housed in single cages at a constant temperature $\left(25^{\circ} \mathrm{C}\right)$ in a clean animal house. Illumination was provided for $12 \mathrm{~h}$ every day; food was provided in the evening, and water was available ad libitum.

\section{Experimental parameters measured Common parameters}

Plasma glucose: Plasma glucose was determined using the glucose oxidation method (One Touch, Ultra Easy Glucometer, LifeScan), as previously described [21].

Plasma insulin: Prior to sacrifice of the animal, a $1 \mathrm{~mL}$ blood sample was withdrawn from the heart of each rat, and stored at $-20^{\circ} \mathrm{C}$ after centrifugation. Blood insulin was determined with a radioimmunoassay (RIA) kit purchased from the Beijing Atomic Energy Research Institute, Beijing, China, using a DFM 96-type multi-tube radioimmunoassay counter (ZhongCheng Mechanical and Electronic Technology Development Co., Ltd, Hefei City, Anhui Province, China). The intra-array coefficient of variation was $<2.5 \%$, while the inter-array coefficient of variation was $<3.5 \%$.

\section{Index of insulin resistance}

Insulin resistance was expressed as the homeostasis model assessment-insulin resistance (HOMA-IR):

HOMA-IR $=$ insulin $(\mathrm{mIU} / \mathrm{L}) \times$ glucose $(\mathrm{mmol} / \mathrm{L}) / 22.5$ [22]

\section{Determination of CSF insulin}

CSF insulin level was determined according to the method of Hoistad et al. [23]. Rats were anesthetized with $20 \%$ ethyl carbamate (urethane). The skin of the head was cut away, and the subcutaneous tissue dissected to unmask the cerebellum and medulla oblongata. The meninges were tapped with the needle of a syringe, through which a drainage catheter was inserted, and $20-50 \mu \mathrm{L}$ CSF was collected from each rat.

\section{Determination of hippocampal AKT/GSK3 $\beta$ and tau-protein levels}

After the rat had been sacrificed, the hippocampus was removed and homogenized on ice $\left(12000 \times \mathrm{g}\right.$ for $10 \mathrm{~min}$ at $\left.4^{\circ} \mathrm{C}\right)$ in a pro- 


\begin{tabular}{|llll|}
\hline Group & Type 2 diabetes group (T2D) & Control group (CTL) & Pioglitazone group (PIO) \\
Number & 5 & 7 & 6 \\
\hline Diet & $\begin{array}{l}\text { High glucose, high fat, } \\
\text { high protein diet }\end{array}$ & Normal diet & $\begin{array}{l}\text { High glucose, high fat, } \\
\text { high protein diet }\end{array}$ \\
\hline STZ injection & Yes & No & Yes \\
\hline Weight before sacrifice $(\mathrm{kg})$ & $436.8 \pm 11.82^{* * *}$ & $314.4 \pm 4.45$ & $444.6 \pm 6.87^{* * *}$ \\
\hline Plasma glucose (mmol/L) & $21.5 \pm 7.13^{* * *}$ & $6.54 \pm 1.35$ & $8.84 \pm 0.73^{\# \# \#}$ \\
\hline Plasma insulin (mIU/L) & $27.54 \pm 2.32^{* * *}$ & $9.78 \pm 1.37$ & $12.36 \pm 2.48^{\# \# \#}$ \\
\hline CSF insulin (mIU/L) & $1.18 \pm 0.78^{*}$ & $2.82 \pm 0.24$ & $1.14 \pm 0.42$ \\
\hline HOMA-IR & $28.8 \pm 7.01^{* * *}$ & $2.76 \pm 0.34$ & $4.07 \pm 2.11^{\# \# \#}$ \\
\hline
\end{tabular}

Data are expressed as the mean \pm SD. Statistical comparisons were made using ANOVA with a Bonferroni post-hoc test. " $P<0.05$ and

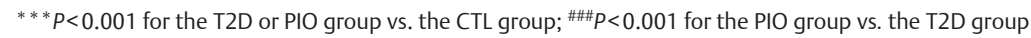

\begin{tabular}{|c|c|c|c|c|}
\hline Antibody & Type & Specificity & $\begin{array}{l}\text { Phosphorylation } \\
\text { sites }\end{array}$ & Reference/Source \\
\hline GSK-3 $\beta$ & Poly & Total GSK-3 $\beta$ & & Biovision, Mountain View, CA \\
\hline p-GSK-3 $\beta$ & Poly & p-GSK-3ß & Ser9 & Cell Signaling, Danvers, MA \\
\hline AKT (ab8805) & Poly & Total AKT & & Abcam, Cambridge, MA \\
\hline p-AKT (ab38449) & Poly & p-AKT & Thr308 & Abcam, Cambridge, MA \\
\hline $\mathrm{H}-71$ & Poly & $\alpha$ fragment of insulin receptor & & Santa Cruz, Santa Cruz, CA \\
\hline $\mathrm{H}-70$ & Poly & $\beta$ fragment of insulin receptor & & Santa Cruz, Santa Cruz, CA \\
\hline $\operatorname{Actin}(\mathrm{I}-19)$ & Poly & $\beta$-actin & & Santa Cruz, Santa Cruz, CA \\
\hline
\end{tabular}

Table 2 Antibodies employed in this study.

Table 1 Characteristics of the rats in the 3 experimental groups. tein extraction solution $(40 \mathrm{mmol} / \mathrm{L}$ Tris- $\mathrm{HCl}, \mathrm{pH} 7.0,1 \%$ Triton $\mathrm{X}-100,0.2 \%$ sodium dodecyl sulfate [SDS], $1.0 \mathrm{mmol} / \mathrm{L}$ sodium deoxycholate, $1.0 \mathrm{mmol} / \mathrm{L} \mathrm{Na}_{3} \mathrm{VO}_{4}, 50 \mathrm{mmol} / \mathrm{L} \mathrm{NaF}, 1.0 \mathrm{mmol} / \mathrm{L}$ phenylmethylsulfonyl fluoride [PMSF], $2.0 \mathrm{mg} / \mathrm{L}$ aprotinin, $2.0 \mathrm{mg} / \mathrm{L}$ leupeptin, $2.0 \mathrm{mg} / \mathrm{L}$ pepstatin, $1.0 \mathrm{mmol} / \mathrm{L}$ ethylene glycol tetraacetic acid [EGTA] and $1.0 \mathrm{mmol} / \mathrm{L}$ ethylene diamine tetraacetic acid [EDTA]). The supernatant was taken to determine protein concentration using the Bradford method, and the remainder was stored at $-80^{\circ} \mathrm{C}$ for later use in western blot experiments.

For western blot experiments, the frozen sample was thawed, mixed with $2 \times$ buffer solution and denatured at $100^{\circ} \mathrm{C}$ for $5 \mathrm{~min}$. $10-30 \mu g$ protein was added into each lane of a vertical electrophoresis chamber for electrophoresis on a $10 \%$ SDS polyacrylamide gel. After electrophoresis, protein was transferred onto a nitrocellulose (NC) membrane, shaken with $5 \%$ bovine serum albumin (BSA) for $2 \mathrm{~h}$, and then hybridized overnight (at $4^{\circ} \mathrm{C}$ ) with the primary antibody (all antibodies used are listed in $\triangle$ Table 2). The NC membrane was subsequently washed 3 times with phosphate buffered saline with Tween-20 (PBST), $10 \mathrm{~min}$ for each wash, and then hybridized with horseradish peroxidase-labeled secondary antibody (goat anti-rabbit IgG, goat anti-mouse IgG or rabbit anti-goat IgG, as appropriate; antibodies purchased from Pierce Co.) for $1 \mathrm{~h}$, with shaking. Following this, the membrane was washed 3 times with PBST (10 min for each wash), and the bands visualized with enhanced chemiluminescence (ECL) and developed on film. Immunoreactive bands were quantitatively analyzed using BandScan v5.0 software.

\section{Statistical analysis}

Data were analyzed using the Prism 5.0 software package. Measured data are expressed as means \pm standard deviations (SD). Comparison of the means between groups was carried out using analysis of variance (ANOVA) with a Bonferroni post-hoc test. A value of $P<0.05$ was taken to be indicative of statistical significance.

\section{Results}

$\nabla$

Blood glucose, insulin, insulin resistance index and CSF insulin levels in the 3 groups of rats

As shown in $\odot$ Table 1, the plasma glucose level and the plasma insulin level in the T2D group were both significantly higher than the corresponding values in the CTL group, whereas the CSF insulin level in the T2D group was significantly lower than that in the CTL group. The plasma insulin and glucose levels in the PIO group were significantly lower than the corresponding values in the T2D group, but not significantly different from those in the CTL group. The CSF insulin level in the PIO group was not significantly different from that in the T2D group, but was significantly lower than that in the CTL group.

HOMA-IR in the T2D group was significantly higher than that in the CTL group, while HOMA-IR in the PIO group was significantly lower than that in the T2D group, and not significantly different from that in the CTL group ( $\bullet$ Table 1 ).

\section{Hippocampal levels of total and phosphorylated AKT/ GSK3 $\beta$ and tau-protein}

As shown in $\odot$ Fig. 1 and $\odot$ Fig. 2, hippocampal levels of total AKT, total GSK-3 $\beta$ and total tau-protein in the T2D group were not significantly different from the corresponding values in the CTL group. However, the level of AKT phosphorylated at amino acid residue Thr308 in the T2D group was significantly lower than that in the CTL group. In addition, the level of GSK-3 $\beta$ phosphorylated at amino acid residue Ser9 in the T2D group was significantly lower than that in the CTL group, while the level of tau-protein phosphorylated at amino acid residues Ser199/ Ser396 in the T2D group was significantly higher than that in the CTL group.

Hippocampal levels of total AKT, total GSK-3 $\beta$ and total tau-protein in the PIO group were not significantly different from the corresponding values in the T2D group. However, the levels of Thr308-phosphorylated AKT and Ser9-phosphorylated GSK-3 $\beta$ in the PIO group were significantly higher than those in the T2D group, while the level of Ser199/Ser396-phosphorylated tau- 


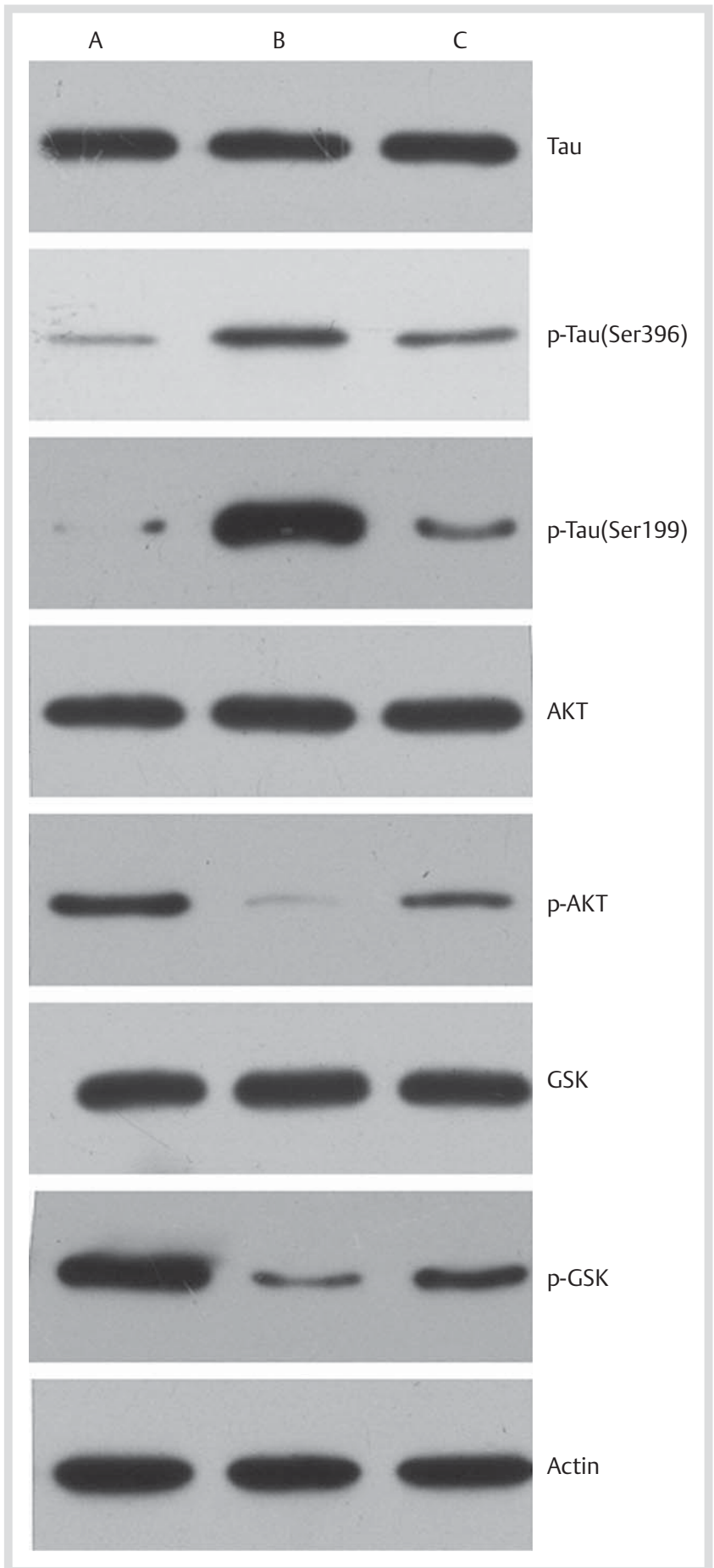

Fig. 1 Western blot analyses of tau-protein, AKT and GSK. Crude hippocampal extracts $(10-30 \mu \mathrm{g} /$ lane) were analyzed using the western blot technique. An actin blot was included as a loading control. A, CTL group; B, T2D group; C, PIO group.

protein in the PIO group was significantly lower than that in the T2D group.

\section{Discussion}

Insulin has different effects in the brain to those it has in peripheral tissues, but as in the periphery, it exerts effects through actions at the insulin receptor (IR) [24]. After insulin binds with the IR, the IR is autophosphorylated at tyrosine residues, leading to a signaling cascade that involves phosphorylation of numerous intracellular targets, including insulin receptor substrate (IRS), PI3K/AKT (activation) and GSK-3 $\beta$ (depression). The outcome of insulin binding to the IR is the promotion of insulinsensitive GLUT (glucose transporter) translocation and a series of physiologic processes in favor of normal neural function, including inhibition of tau-protein hyperphosphorylation and $A \beta$ accumulation, both of which are risk factors for AD.

Our previous study suggested that rats with either type 1 or type 2 diabetes demonstrated increased hippocampal GSK-3 $\beta$ activity and tau-protein hyperphosphorylation [25]. This indicated that both insulin deficiency and insulin resistance are associated with tau-protein hyperphosphorylation. However, it was not clear whether the intracerebral insulin level was altered in rats with type 2 diabetes, and whether an insulin sensitizer could normalize the intracerebral insulin level and insulin signal transduction pathway, and thus decrease tau-protein hyperphosphorylation.

In the present study, we found that rats with T2D showed decreased CSF insulin levels, decreased hippocampal AKT activity, increased GSK-3 $\beta$ activity and hyperphosphorylation of tauprotein. Taken together, these data suggest that the brains of rats with type 2 diabetes show both insulin resistance and insulin deficiency, resulting in tau-protein hyperphosphorylation. Since brain insulin is derived from peripheral blood, its deficiency may be the result of disruption of the BBB by chronic hyperglycemia.

Pioglitazone is an oral anti-diabetic agent that acts primarily by decreasing insulin resistance through its action at PPAR r receptors in tissues such as adipose tissue, skeletal muscle and the liver. Activation of PPAR nuclear receptors modulates the transcription of a number of insulin responsive genes involved in the control of glucose and lipid metabolism [26]. It was interesting to note that, in our study, treatment with pioglitazone resulted in a reduction in the plasma insulin concentration. We speculate that the pioglitazone-mediated improvement in the insulin sensitivity led to a restoration of plasma glucose to normal (control) levels that, in turn, resulted in a fall in plasma insulin to a level similar to that of the control. This is consistent with previous studies reporting that pioglitazone was able to reduce fasting plasma insulin levels, both in rats fed a high-fat diet [27], and in patients with AD [18] or T2D [28-30].

After pioglitazone treatment, the CSF insulin level was not altered in rats with T2D, but hippocampal AKT activity was increased, GSK-3 $\beta$ activity was decreased, and tau-protein hyperphosphorylation was reduced. This implies that an insulin sensitizer can ameliorate intracerebral insulin resistance and reduce tau-protein hyperphosphorylation, despite a lack of effect on the CSF insulin level.

\section{Acknowledgments}

$\nabla$

The study was supported by the Natural Science Foundation of China (NSFC) (81100582), China International Medical Foundation (CIMF) - Novo Nordisk China Diabetes Young Scientific Talent Research Funding (2012041), and the General Project of Hubei Province Health Department (JX5B04). 


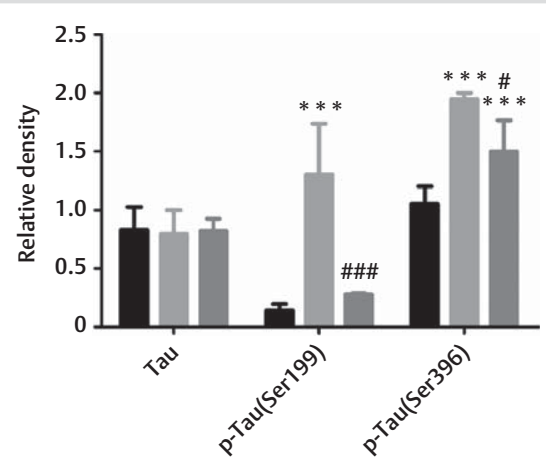

Insulin signal transduction in rat hippocampus a CTL T2D Pioglitazone

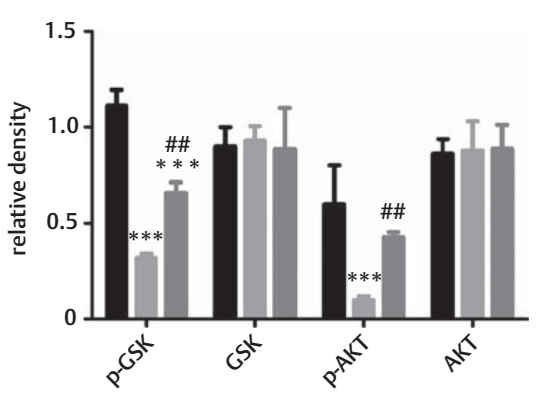

Insulin signal transduction in rat hippocampus - CTL T2D Pioglitazone
Fig. 2 Western blot analyses of kinases, in the rat hippocampus, that are involved in the insulin signal transduction pathway. Blots such as those shown in Fig. 1 were quantitated densitometrically. All data are presented as the means \pm SDs of the relative immunoreactivities. ${ }^{* *} P<0.001$ for the T2D or PIO group vs. the CTL group; ${ }^{\#} P<0.05$.

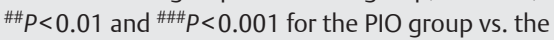
T2D group.

\section{Conflict of interest: None.}

\section{References}

1 Yang $W$, Lu J, Weng $J$ et al. Prevalence of diabetes among men and women in China. N Engl J Med 2010; 362: 1090-1101

2 Alzheimer's Association. 2010; 2010 Alzheimer's disease facts and figures. Alzheimers Dement 2010; 6: 158-194

3 Arvanitakis Z, Wilson RS, Bienias JL et al. Diabetes mellitus and risk of Alzheimer disease and decline in cognitive function. Arch Neurol 2004; 61: 661-666

4 Xu WL, Qiu CX, Wahlin A et al. Diabetes mellitus and risk of dementia in the Kungsholmen project: a 6-year follow-up study. Neurology 2004; 63: 1181-1186

5 de la Monte SM. Contributions of brain insulin resistance and deficiency in amyloid-related neurodegeneration in Alzheimer's disease. Drugs 2012; 72: 49-66

6 Plaschke K, Kopitz J, Siegelin $M$ et al. Insulin-resistant brain state after intracerebroventricular streptozotocin injection exacerbates Alzheimer-like changes in Tg2576 AbetaPP-overexpressing mice. J Alzheimers Dis 2010; 19: 691-704

7 Belgardt BF, Bruning JC. CNS leptin and insulin action in the control of energy homeostasis. Ann N Y Acad Sci 2010; 1212: 97-113

8 Craft S, Peskind E, Schwartz MW et al. Cerebrospinal fluid and plasma insulin levels in Alzheimer's disease: relationship to severity of dementia and apolipoprotein E genotype. Neurology 1998; 50: 164-168

9 Laron Z. Insulin and the brain. Arch Physiol Biochem 2009; 115: 112-116

10 Park CR, Seeley RJ, Craft $S$ et al. Intracerebroventricular insulin enhances memory in a passive-avoidance task. Physiol Behav 2000; 68: $509-514$

11 Wang X, Zheng $W$, Xie JW et al. Insulin deficiency exacerbates cerebral amyloidosis and behavioral deficits in an Alzheimer transgenic mouse model. Mol Neurodegener 2010; 5: 46

12 Talbot K, Wang HY, Kazi $H$ et al. Demonstrated brain insulin resistance in Alzheimer's disease patients is associated with IGF-1 resistance, IRS-1 dysregulation, and cognitive decline. J Clin Invest 2012; 122: 1316-1338

13 de la Monte SM. Brain insulin resistance and deficiency as therapeutic targets in Alzheimer's disease. Curr Alzheimer Res 2012; 9: 35-66

14 Williamson R, McNeilly A, Sutherland C. Insulin resistance in the brain: an old-age or new-age problem? Biochem Pharmacol 2012; 84: 737-745

15 Li X, Pan W, Yang GZ et al. Proteome analysis of differential protein expression in brain of rats with type 1 diabetes mellitus. Exp Clin Endocrinol Diabetes 2011; 119: 265-270
16 Qian W, Shi J, Yin X et al. PP2A regulates tau phosphorylation directly and also indirectly via activating GSK-3beta. J Alzheimers Dis 2010; 19: 1221-1229

17 Zhang XJ, Yang SS, Zhang MX. Common pathogenesis of type 2 diabetes and Alzheimer's disease: a potential mechanism. Chin J Pathophysiol 2010; 26: 1107-1114

18 Sato T, Hanyu H, Hirao $K$ et al. Efficacy of PPAR-gamma agonist pioglitazone in mild Alzheimer disease. Neurobiol Aging 2011; 32: 1626-1633

19 Kanazawa I, Yamamoto M, Yamaguchi T et al. Effects of metformin and pioglitazone on serum pentosidine levels in type 2 diabetes mellitus. Exp Clin Endocrinol Diabetes 2011; 119: 362-365

20 Dorafshar AH, Moodley K, Khoe $M$ et al. Pioglitazone improves superoxide dismutase mediated vascular reactivity in the obese Zucker rat. Diab Vasc Dis Res 2010; 7: 20-27

21 Trinder $P$. Determination of glucose in blood using glucose oxidase with an alternative oxygen acceptor. Ann Clin Biochem 1969; 6: 24-27

22 Matthews DR, Hosker JP, Rudenski AS et al. Homeostasis model assessment: insulin resistance and beta-cell function from fasting plasma glucose and insulin concentrations in man. Diabetologia 1985; 28: 412-419

23 Höistad M, Samskog J, Jacobsen KX et al. Detection of beta-endorphin in the cerebrospinal fluid after intrastriatal microinjection into the rat brain. Brain Res 2005; 1041: 167-180

24 Leavens KF, Birnbaum MJ. Insulin signaling to hepatic lipid metabolism in health and disease. Crit Rev Biochem Mol Biol 2011; 46: 200-215

$25 \mathrm{Hu}$ SH, Yang Y, Ai DY. Tau protein phosphorylation levels and glycogen synthase kinase- $3 \beta$ activities were increased in hippocampus of rats with type 1 or type 2 diabetes. Basic Clinic Med 2007; 27: 1001-1005

26 Panunti B, Fonseca $V$. Effects of PPAR gamma agonists on cardiovascular function in obese, non-diabetic patients. Vascul Pharmacol 2006; 45: 29-35

27 Srinivasan K, Patole PS, Kaul CL et al. Reversal of glucose intolerance by by pioglitazone in high fat diet-fed rats. Methods Find Exp Clin Pharmacol 2004; 26: 327-333

28 Teramoto T, Yamada N, Shirai $K$ et al. Effects of pioglitazone hydrochloride on Japanese patients with type 2 diabetes mellitus. J Atheroscler Thromb 2007; 14: 86-93

29 Pavo I, Jermendy G, Varkonyi TT et al. Effect of pioglitazone compared with metformin on glycemic control and indicators of insulin sensitivity in recently diagnosed patients with type 2 diabetes. J Clin Endocrinol Metab 2003; 88: 1637-1645

30 Kubo K. Effect of pioglitazone on blood proinsulin levels in patients with type 2 diabetes mellitus. Endocr J 2002; 49: 323-328 